\title{
Oscillation of second-order difference equations
}

\author{
Ying Huang ${ }^{a, b}$, Jingjing Wang ${ }^{c}$, Tongxing $\mathrm{Li}^{\mathrm{d}, \mathrm{e}, *}$ \\ a School of Mathematics, Jilin University, Changchun, Jilin 130012, P. R. China. \\ ${ }^{b}$ School of Mathematics and System Science, Shenyang Normal University, Shenyang, Liaoning 110034, P. R. China. \\ ${ }^{c}$ School of Information Science \& Technology, Qingdao University of Science \& Technology, Qingdao, Shandong 266061, P. R. China. \\ ${ }^{d}$ LinDa Institute of Shandong Provincial Key Laboratory of Network Based Intelligent Computing, Linyi University, Linyi, Shandong \\ 276005, P. R. China. \\ ${ }^{e}$ School of Informatics, Linyi University, Linyi, Shandong 276005, P. R. China.
}

Communicated by $\mathrm{X}$. Qin

\begin{abstract}
We obtain new oscillation theorems for a class of second-order linear difference equations. Our criteria complement and improve related results reported in the literature. An illustrative example is given. (c)2017 All rights reserved.
\end{abstract}

Keywords: Oscillation, second-order, difference equation.

2010 MSC: 39A21.

\section{Introduction}

In this paper, we are concerned with the oscillation of a linear second-order difference equation

$$
\Delta^{2} x_{n-1}+p_{n} x_{n}=0, \quad n=0,1,2, \ldots,
$$

where $\Delta$ is the forward difference operator satisfying $\Delta x_{n}=x_{n+1}-x_{n}$ and $\left\{p_{n}\right\}$ is a sequence of nonnegative real numbers. A solution $\left\{x_{n}\right\}$ of (1.1) is termed oscillatory if it is neither eventually positive nor eventually negative; otherwise, it is called nonoscillatory. Equation (1.1) is said to be oscillatory if all its nontrivial solutions oscillate.

Oscillation and asymptotic behavior of various classes of difference equations have always attracted interest of researchers; see, e.g., the monograph [1], the papers [2-16], and the references cited therein. In particular, several interesting oscillation results for equation (1.1) were established in the papers by Erbe and Zhang [4], Jiang and Li [5], Lei [6], Sun [8], and Zhang and Cheng [14], some of which we present below for the convenience of the reader. In the following, we use the notation:

$$
u_{n}(\alpha)=n^{1-\alpha} \sum_{k=n+1}^{\infty} k^{\alpha} p_{k}, \quad \sum_{k=1}^{\infty} k^{\alpha} p_{k}<\infty, \quad p_{*}(\alpha)=\liminf _{n \rightarrow \infty} u_{n}(\alpha), \quad \text { and } \quad p^{*}(\alpha)=\limsup _{n \rightarrow \infty} u_{n}(\alpha) .
$$

\footnotetext{
*Corresponding author

Email address: litongx2007@163.com (Tongxing Li)
} 
Theorem 1.1 ([4]). If

$$
\liminf _{n \rightarrow \infty} n^{2} p_{n} \geqslant \frac{1}{4}
$$

then equation (1.1) is oscillatory.

Theorem 1.2 ([14]). If

$$
p_{*}(0)>\frac{1}{4}
$$

then equation (1.1) is oscillatory.

Theorem $1.3([5,6])$. Let $p_{*}(0) \leqslant 1 / 4$. If there exists a constant $\alpha>1$ such that

$$
p^{*}(\alpha)>\frac{\alpha^{2}}{4(\alpha-1)}-\frac{1}{2}\left(1-\sqrt{1-4 p_{*}(0)}\right),
$$

then equation (1.1) is oscillatory.

This study was strongly motivated by the research of Erbe and Zhang [4], Jiang and Li [5], Lei [6], and Zhang and Cheng [14]. Its purpose is to obtain new oscillation criteria for equation (1.1) that improve Theorems 1.1 and 1.2 and complement Theorem 1.3. It is not difficult to see that if there exists a constant $\alpha<1$ such that

$$
\sum_{k=1}^{\infty} k^{\alpha} p_{k}=\infty
$$

then equation (1.1) is oscillatory. In the sequel, we assume that

$$
\sum_{k=1}^{\infty} k^{\alpha} p_{k}<\infty, \quad \alpha<1
$$

As usual, all functional inequalities considered in this paper are supposed to hold eventually. Without loss of generality, we deal only with positive solutions of (1.1) since $\left\{-x_{n}\right\}$ is also a solution of this equation provided that $\left\{x_{n}\right\}$ is a solution.

\section{Lemmas}

To prove the main results, we need the following lemmas. For a compact presentation of our results, we adopt the notation:

$$
q=\liminf _{n \rightarrow \infty} \frac{1}{n} \sum_{k=1}^{n} k^{2} p_{k}, \quad w_{n}=\frac{\Delta x_{n-1}}{x_{n-1}}, \quad r=\liminf _{n \rightarrow \infty} n w_{n+1}, \quad \text { and } \quad R=\limsup _{n \rightarrow \infty} n w_{n+1} .
$$

Lemma 2.1. If $\alpha \in[0,1)$, then

$$
\sum_{k=n+1}^{\infty} \frac{\left(\Delta k^{\alpha}\right)^{2}}{k^{\alpha}} \leqslant \frac{\alpha^{2}}{1-\alpha} n^{\alpha-1}
$$

and

$$
\sum_{k=n+1}^{\infty} k^{\alpha-2}<\frac{n^{\alpha-1}}{1-\alpha}
$$

Proof. By virtue of the mean value theorem, there exist two numbers $\xi_{k} \in(k, k+1)$ and $\eta_{k} \in(k-1, k)$ such that

$$
\frac{\left(\Delta k^{\alpha}\right)^{2}}{k^{\alpha}}=\frac{\alpha^{2} \xi_{k}^{2 \alpha-2}}{k^{\alpha}} \leqslant \frac{\alpha^{2} k^{2 \alpha-2}}{k^{\alpha}} \text { and } \frac{\Delta(k-1)^{1-\alpha}}{1-\alpha}=\eta_{k}^{-\alpha} \geqslant \frac{1}{k^{\alpha}} .
$$


Hence, we deduce that

$$
\sum_{k=n+1}^{\infty} \frac{\left(\Delta k^{\alpha}\right)^{2}}{k^{\alpha}} \leqslant \frac{\alpha^{2}}{1-\alpha} \sum_{k=n+1}^{\infty} \frac{\Delta(k-1)^{1-\alpha}}{k^{2-2 \alpha}} .
$$

Define $r(t)=(k-1)^{1-\alpha}+(t-k+1) \Delta(k-1)^{1-\alpha}, k-1 \leqslant t \leqslant k$. Then $r^{\prime}(t)=\Delta(k-1)^{1-\alpha},(k-1)^{1-\alpha} \leqslant$ $r(t) \leqslant k^{1-\alpha}, k-1 \leqslant t \leqslant k$, and so

$$
\frac{\Delta(k-1)^{1-\alpha}}{k^{2-2 \alpha}}=\int_{k-1}^{k} \frac{\Delta(k-1)^{1-\alpha}}{k^{2-2 \alpha}} d t \leqslant \int_{k-1}^{k} \frac{r^{\prime}(t)}{r^{2}(t)} d t=\frac{1}{(k-1)^{1-\alpha}}-\frac{1}{k^{1-\alpha}} .
$$

It follows from the latter inequality and (2.3) that (2.1) holds. Using the inequality

$$
\sum_{k=n+1}^{\infty} \frac{1}{k^{2-\alpha}}<\int_{n}^{\infty} \frac{1}{t^{2-\alpha}} d t
$$

we have (2.2). The proof is complete.

Lemma 2.2 ([5]). Let $\left\{x_{n}\right\}$ be a nonoscillatory solution of equation (1.1) such that $x_{n-1}>0$ for $n \geqslant n_{0}$. Then

$$
\begin{gathered}
\Delta w_{n}+w_{n} w_{n+1}+p_{n} \leqslant 0, \quad n \geqslant n_{0}, \\
w_{n} \geqslant w_{n+1}, \quad 0 \leqslant\left(n-n_{0}\right) w_{n}<1, \quad n \geqslant n_{0},
\end{gathered}
$$

and

$$
p_{*}(0) \leqslant r-r^{2}, \quad q \leqslant R-R^{2} .
$$

\section{Main results}

Let

$$
M_{1}=\frac{1}{2}(1+\sqrt{1-4 q}) \text { and } \quad M_{2}=\frac{1}{2}\left(1-\sqrt{1-4 p_{*}(0)}\right) .
$$

We give the following oscillation results for equation (1.1).

Theorem 3.1. Let $\mathrm{q} \leqslant 1 / 4$. If there exists a constant $\alpha \in[0,1)$ such that

$$
p^{*}(\alpha)>\frac{\alpha^{2}}{4(1-\alpha)}+M_{1}
$$

then equation (1.1) is oscillatory.

Proof. Let $\left\{x_{n}\right\}$ be a nonoscillatory solution of equation (1.1) such that $x_{n-1}>0$ for $n \geqslant n_{0}$. From (2.4) and (2.5), we conclude that

$$
p_{k} \leqslant-\Delta w_{k}-w_{k+1}^{2} \text {. }
$$

Multiplying (3.2) by $k^{\alpha}$ and summing the resulting inequality from $n+1$ to $\infty$, we get

$$
\begin{aligned}
\sum_{k=n+1}^{\infty} k^{\alpha} p_{k} & \leqslant-\sum_{k=n+1}^{\infty} k^{\alpha} \Delta w_{k}-\sum_{k=n+1}^{\infty} k^{\alpha} w_{k+1}^{2} \\
& =(n+1)^{\alpha} w_{n+1}+\sum_{k=n+1}^{\infty} w_{k+1} \Delta k^{\alpha}-\sum_{k=n+1}^{\infty} k^{\alpha} w_{k+1}^{2} .
\end{aligned}
$$

Using (3.3), we have

$$
\sum_{k=n+1}^{\infty} k^{\alpha} p_{k} \leqslant(n+1)^{\alpha} w_{n+1}+\frac{1}{4} \sum_{k=n+1}^{\infty} \frac{\left(\Delta k^{\alpha}\right)^{2}}{k^{\alpha}}-\sum_{k=n+1}^{\infty}\left(k^{\frac{\alpha}{2}} w_{k+1}-\frac{1}{2} k^{-\frac{\alpha}{2}} \Delta k^{\alpha}\right)^{2}
$$




$$
\leqslant(n+1)^{\alpha} w_{n+1}+\frac{1}{4} \sum_{k=n+1}^{\infty} \frac{\left(\Delta k^{\alpha}\right)^{2}}{k^{\alpha}}
$$

which yields

$$
\limsup _{n \rightarrow \infty} n^{1-\alpha} \sum_{k=n+1}^{\infty} k^{\alpha} p_{k} \leqslant \limsup _{n \rightarrow \infty}\left(\frac{n+1}{n}\right)^{\alpha} n w_{n+1}+\limsup _{n \rightarrow \infty} \frac{1}{4} n^{1-\alpha} \sum_{k=n+1}^{\infty} \frac{\left(\Delta k^{\alpha}\right)^{2}}{k^{\alpha}} .
$$

Hence, by (2.1) and (2.5), we deduce that

$$
p^{*}(\alpha) \leqslant \limsup _{n \rightarrow \infty} n w_{n+1}+\limsup _{n \rightarrow \infty} \frac{1}{4} n^{1-\alpha} n^{\alpha-1} \frac{\alpha^{2}}{1-\alpha}=R+\frac{\alpha^{2}}{4(1-\alpha)} .
$$

On the other hand, we have

$$
\mathrm{R} \leqslant \mathrm{M}_{1}
$$

due to (2.6). Therefore, we arrive at

$$
p^{*}(\alpha) \leqslant \frac{\alpha^{2}}{4(1-\alpha)}+\frac{1}{2}(1+\sqrt{1-4 q}),
$$

which contradicts (3.1). The proof is complete.

Theorem 3.2. Let $\mathrm{p}_{*}(0) \leqslant 1 / 4$ and $\mathrm{q} \leqslant 1 / 4$. If there exists a constant $\alpha \in\left[\mathrm{M}_{2}, 1\right)$ such that

$$
p^{*}(\alpha)>\frac{M_{1}\left(1-M_{2}\right)}{1-\alpha},
$$

then equation (1.1) is oscillatory.

Proof. Assume that $\left\{x_{n}\right\}$ is a positive solution of equation (1.1) such that $x_{n-1}>0$ for $n \geqslant n_{0}$. By virtue of (2.6),

$$
r \geqslant M_{2}
$$

From the latter inequality and (3.4), we conclude that, for any $\varepsilon>0$, there exists an $n_{1} \geqslant n_{0}$ such that

$$
M_{2}-\varepsilon<n w_{n+1} \leqslant\left(\frac{n+1}{n}\right)^{\alpha} n w_{n+1}<M_{1}+\varepsilon
$$

for $n \geqslant n_{1}$. On the other hand, as in the proof of Theorem 3.1, we have (3.3). Using the fact that $\Delta \mathrm{k}^{\alpha}=(\mathrm{k}+1)^{\alpha}-\mathrm{k}^{\alpha}<\alpha \mathrm{k}^{\alpha-1}$ and multiplying (3.3) by $\mathrm{n}^{1-\alpha}$, we obtain

$$
\begin{aligned}
n^{1-\alpha} \sum_{k=n+1}^{\infty} k^{\alpha} p_{k} & \leqslant\left(\frac{n+1}{n}\right)^{\alpha} n w_{n+1}+n^{1-\alpha} \sum_{k=n+1}^{\infty} k^{\alpha-2}\left[k w_{k+1}\left(\alpha-k w_{k+1}\right)\right] \\
& \leqslant M_{1}+\varepsilon+\left(M_{1}+\varepsilon\right)\left(\alpha+\varepsilon-M_{2}\right) n^{1-\alpha} \sum_{k=n+1}^{\infty} k^{\alpha-2} .
\end{aligned}
$$

Substituting (2.2) into the latter inequality, we deduce that

$$
n^{1-\alpha} \sum_{k=n+1}^{\infty} k^{\alpha} p_{k} \leqslant\left(M_{1}+\varepsilon\right)\left[1+n^{1-\alpha} \frac{n^{\alpha-1}}{1-\alpha}\left(\alpha+\varepsilon-M_{2}\right)\right]=\left(M_{1}+\varepsilon\right) \frac{1-M_{2}+\varepsilon}{1-\alpha} .
$$

Since $\varepsilon>0$ is arbitrary, we get

$$
p^{*}(\alpha) \leqslant \frac{M_{1}\left(1-M_{2}\right)}{1-\alpha},
$$

which contradicts (3.5). This completes the proof. 
Remark 3.3. Observe that $M_{2} \in[0,1 / 2]$ in the case when $p_{*}(0) \in[0,1 / 4]$. Let all hypotheses of Theorem 3.2 be satisfied with condition $\alpha \in\left[M_{2}, 1\right)$ replaced by $\alpha \in[1 / 2,1)$. Then equation (1.1) is oscillatory.

Remark 3.4. Note that $\alpha>1$ is required in Theorem 1.3. Hence, Theorems 3.1 and 3.2 complement the results obtained in $[5,6]$.

\section{Example}

Example 4.1. Consider the difference equation

$$
\Delta^{2} x_{n-1}+p_{n} x_{n}=0, \quad n=0,1,2, \ldots,
$$

where

$$
p_{n}=\left\{\begin{array}{ll}
\frac{1}{6^{m}}, & n=6^{m}, \\
0, & n \neq 6^{m},
\end{array} \quad m=0,1,2, \ldots\right.
$$

It is not difficult to verify that

$$
p_{*}(0)=\liminf _{n \rightarrow \infty} u_{n}(0)=\frac{1}{5}<\frac{1}{4} \quad \text { and } \quad q=\limsup _{n \rightarrow \infty} \frac{1}{n} \sum_{k=1}^{n} k^{2} p_{k}=\frac{1}{5}<\frac{1}{4} .
$$

Thus, we conclude that

$$
M_{1}=\frac{1}{2}(1+\sqrt{1-4 q})=\frac{1}{2}\left(1+\frac{\sqrt{5}}{5}\right) \quad \text { and } \quad M_{2}=\frac{1}{2}\left(1-\sqrt{1-4 p_{*}(0)}\right)=\frac{1}{2}\left(1-\frac{\sqrt{5}}{5}\right) .
$$

Let $\alpha=1 / 2$. Then

$$
\frac{M_{1}\left(1-M_{2}\right)}{1-\alpha}=\frac{1}{2}\left(\frac{6}{5}+\frac{2 \sqrt{5}}{5}\right) \quad \text { and } \quad p^{*}(\alpha)=\limsup _{n \rightarrow \infty} u_{n}\left(\frac{1}{2}\right)=\limsup _{n \rightarrow \infty} n^{\frac{1}{2}} \sum_{k=n+1}^{\infty} k^{\frac{1}{2}} p_{k}=\frac{6+\sqrt{6}}{5},
$$

and so

$$
p^{*}(\alpha)>\frac{M_{1}\left(1-M_{2}\right)}{1-\alpha} .
$$

Therefore, by Theorem 3.2, equation (4.1) is oscillatory. Observe that Theorems 1.1 and 1.2 cannot be applied to equation (4.1). Hence, Theorem 3.2 improves Theorems 1.1 and 1.2.

\section{Acknowledgment}

The authors express their sincere gratitude to the editors and anonymous referee for the careful reading of the original manuscript and useful comments that helped to improve the presentation of the results and accentuate important details. This research is supported by NNSF of P. R. China (Grant Nos. 61503171, 61304222, and 11626161), CPSF (Grant Nos. 2015M582091 and 2014M551905), NSF of Shandong Province (Grant No. ZR2016JL021), DSRF of Linyi University (Grant No. LYDX2015BS001), and the AMEP of Linyi University, P. R. China.

\section{References}

[1] R. P. Agarwal, M. Bohner, S. R. Grace, D. O'Regan, Discrete oscillation theory, Hindawi Publishing Corporation, New York, (2005). 1

[2] R. P. Agarwal, M. Bohner, T.-X. Li, C.-H. Zhang, Oscillation criteria for second-order dynamic equations on time scales, Appl. Math. Lett., 31 (2014), 34-40. 1

[3] S. S. Cheng, T. C. Yan, H. J. Li, Oscillation criteria for second order difference equation, Funkcial. Ekvac., 34 (1991), 223-239. 
[4] L. H. Erbe, B. G. Zhang, Oscillation of second order linear difference equations, Chinese J. Math., 16 (1988), 239-252. 1, $1.1,1$

[5] J.-C. Jiang, X.-P. Li, Oscillation criteria for second-order linear difference equations, Appl. Math. Comput., 145 (2003), 591-601. 1, 1.3, 1, 2.2, 3.4

[6] C.-M. Lei, Remarks on oscillation of second-order linear difference equation, Appl. Math. Comput., 215 (2009), $2855-$ 2857. 1, 1.3, 1, 3.4

[7] S. Selvarangam, S. Geetha, E. Thandapani, Oscillation and asymptotic behavior of third order nonlinear neutral difference equations with mixed type, J. Nonlinear Funct. Anal., 2017 (2017), 17 pages.

[8] Y. G. Sun, Oscillation and non-oscillation for second-order linear difference equations, Appl. Math. Comput., 170 (2005), 1095-1103. 1

[9] Y. G. Sun, F. W. Meng, Nonoscillation and oscillation of second order half-linear difference equations, Appl. Math. Comput., 197 (2008), 121-127.

[10] E. Thandapani, V. Balasubramanian, Some oscillation results for second order neutral type difference equations, Differ. Equ. Appl., 5 (2013), 319-330.

[11] E. Thandapani, V. Balasubramanian, Some new oscillation criteria for nonlinear neutral delay difference equations, J. Nonlinear Funct. Anal., 2014 (2014), 11 pages.

[12] E. Thandapani, S. Selvarangam, R. Rama, M. Madhan, Improved oscillation criteria for second order nonlinear delay difference equations with non-positive neutral term, Fasc. Math., 56 (2016), 155-165.

[13] J.-L. Yao, F.-W. Meng, Asymptotic behavior of solutions of certain higher order nonlinear difference equation, J. Comput. Appl. Math., 205 (2007), 640-650.

[14] B. G. Zhang, S. S. Cheng, Oscillation criteria and comparison theorems for delay difference equations, Fasc. Math., 25 (1995), 13-32. 1, 1.2, 1

[15] B. G. Zhang, Y. Zhou, Oscillation and nonoscillation for second-order linear difference equations, Comput. Math. Appl., 39 (2000), 1-7.

[16] Y. Zhou, B. G. Zhang, Oscillations of delay difference equations in a critical state, Comput. Math. Appl., 39 (2000), 71-80. 1 